\title{
Suporte organizacional, capital social e atitudes inovadoras: Um estudo correlacional em trabalhadores na cidade de Natal-RN
}

Organizational support, social capital and innovative attitudes: A correlational study among workers in the city of Natal-RN

Apoyo organizacional, capital social y actitudes innovadoras: un estudio correlacional entre trabajadores en la ciudad de Natal-RN

\author{
Nilton S. Formiga \\ ORCID: https://orcid.org/0000-0003-4907-9736 \\ Universidade Potiguar, Brasil \\ E-mail: nsformiga@yahoo.com \\ Antonio Jorge Fernandes \\ ORCID: https://orcid.org/0000-0003-0145-017X \\ Universidade Potiguar, Brasil \\ Universidade de Aveiro, Portugal \\ E-mail: afer@ua.pt \\ Bruna G. O. Freire \\ ORCID: https://orcid.org/0000-0002-0879-2570 \\ Universidade Estadual do Ceará, Brasil \\ E-mail: brunafreireolv@gmail.com \\ Juliana Bianca Maia Franco \\ ORCID: https://orcid.org/0000-0002-7566-2688 \\ Universidade Potiguar, Brasil \\ E-mail: juliana_franco_@hotmail.com \\ Heitor César Costa Oliveira \\ ORCID: https://orcid.org/ 0000-0003-4778-6613 \\ Universidade Potiguar, Brasil \\ E-mail: cartoriosbrasil@gmail.com \\ Gleyssielle Lira Prochazka \\ ORCID: https://orcid.org/0000-0001-6083-1306 \\ Universidade Potiguar, Brasil \\ E-mail: gleyssielle.lira@gmail.com \\ Cristienne Gonçalves Pereira Valin \\ ORCID: https://orcid.org/0000-0002-7928-4856 \\ Universidade Potiguar, Brasil \\ E-mail: cris2n@hotmail.com \\ Sara Ruth Araújo Grangeiro \\ ORCID: https://orcid.org/0000-0001-7446-6020 \\ Universidade Ateneu, Brasil \\ E-mail: sararuthmbc@hotmail.com \\ Ionara Dantas Estevam \\ ORCID: https://orcid.org/0000-0002-4123-3244 \\ Universidade Potiguar, Brasil \\ E-mail: ionaradantas@gmail.com
}

\begin{abstract}
Resumo:
A busca por atitudes inovadoras, assim como o incentivo para o desenvolvimento das relações sociais no ambiente de trabalho, vem sendo considerada variáveis mediadoras do sucesso organizacional. Com isso, ações de reconhecimento e valorização acerca das práticas profissionais tem se tornado prática constante no ambiente corporativo, no entanto tem surgido dúvidas sobre os benefícios e reais contribuições para o bem-estar físico e psicológico dos profissionais. Dessa forma, a presente pesquisa tem por objetivo verificar a influência do suporte organizacional sobre o capital social e as atitudes inovadoras dos trabalhadores que atuam em organizações públicas e privadas. Por meio dos instrumentos de pesquisa propostos por Siqueira (1995), Nahapiet e Ghoshal (1998), Fandiño, Formiga e Menezes (2018) sobre suporte organizacional, capital social e atitudes inovadoras, a pesquisa foi aplicada por meio de questionários eletrônicos a 412 trabalhadores brasileiros que atuam em organizações públicas e privadas. O tratamento dos dados ocorreu por meio de análises de discriminação dos itens, representatividade de conteúdo, análise exploratória e confirmatória das medidas em questão. Para isso foram utilizados os softwares
\end{abstract}


SPSS (versão 21.0) para análise dos dados e observou-se que o desenvolvimento de atitudes inovadoras não ocorre de forma única, mas, tem a empresa que oferece suporte, tornando claro a implementação de vínculos entre os profissionais da organização, a construção dos tipos de relacionamentos e a percepção dos trabalhadores acerca da importância dessa interação, conexão, e compartilhamento de experiência.

Palavras-chave: Suporte; Capital social; Inovação; Trabalhadores; Empresas brasileiras.

\begin{abstract}
:
The search for innovative attitudes, as well as the incentive for the development of social relationships in the workplace, have been considered as mediating variables for organizational success. With this, recognition and appreciation actions about professional practices have become a constant practice in the corporate environment, however doubts about the benefits and real contributions to the physical and psychological well-being of professionals have arisen. Thus, this research aims to verify the influence of organizational support on social capital and innovative attitudes of workers who work in public and private organizations. Using the research instruments proposed by Siqueira (1995), Nahapiet and Ghoshal (1998), Fandiño, Formiga and Menezes (2018) on organizational support, social capital and innovative attitudes, the survey was applied through electronic questionnaires to 412 Brazilian workers from public and private organizations. The data treatment occurred through item discrimination analysis, content representation and relevant measures exploratory and confirmatory analysis. For this, SPSS software (version 21.0) was used for data analysis and it was observed that the development of innovative attitudes does not occur in a unique way, but associated with the organizational support that the company offers, making it clear the implementation of links between the organization's professionals, the construction of relationships types and the workers perception about the importance of this interaction, connection, and sharing of experience.
\end{abstract}

Keywords: Support; Share capital; Innovation; Workers; Brazilian companies.

\begin{abstract}
Resumen
La búsqueda de actitudes innovadoras así como el incentivo para el desarrollo de relaciones sociales en el lugar de trabajo, se han considerado variables mediadoras para el éxito organizacional. Con esto, las acciones de reconocimiento y apreciación sobre las prácticas profesionales se han convertido en una práctica constante en el entorno corporativo, sin embargo, han surgido dudas sobre los beneficios y las contribuciones reales al bienestar físico y psicológico de los profesionales. Por lo tanto, esta investigación tiene como objetivo verificar la influencia del apoyo organizacional en el capital social y las actitudes innovadoras de los trabajadores que trabajan en organizaciones públicas y privadas. Utilizando los instrumentos de investigación propuestos por Siqueira (1995), Nahapiet y Ghoshal (1998), Fandiño, Formiga y Menezes (2018) sobre apoyo organizacional, capital social y actitudes innovadoras, la encuesta se aplicó mediante cuestionarios electrónicos a 412 trabajadores brasileños que trabajan en organizaciones públicas y privadas. El tratamiento de los datos se produjo a través del análisis de la discriminación de elementos, la representación del contenido, el análisis exploratorio y confirmatorio de las medidas en cuestión. Para eso, se utilizó el software SPSS (versión 21.0) para el análisis de datos y se observó que el desarrollo de actitudes innovadoras no se produce de una manera única, pero asociado con el soporte organizativo que ofrece la empresa, lo que deja claro la implementación de vínculos entre los profesionales de la organización, la construcción de tipos de relaciones y la percepción de los trabajadores sobre la importancia de esta interacción, conexión y compartir experiencias.
\end{abstract}

Palabras clave: Apoyo; Capital social; Innovación; Trabajadores; Empresas brasileñas.

\title{
1. Introdução
}

A gestão organizacional, geralmente, vincula-se a uma perspectiva econômica, em que a busca contínua pela vantagem competitiva e a utilização de incentivos financeiros eram os meios de motivação ou punição aos trabalhadores considerados uma prática constante (Verstegen, 2011). A utilização de estratégias análogas de gestão para lidar com a heterogeneidade das demandas contemporâneas se tornara cada vez mais limitadas, precisando com isso, reforma e reinvenção das práticas no mercado (Braia, Curral, \& Gomes, 2014).

Nos últimos anos, o mercado vem exigindo que as organizações se tornem cada vez mais inovadoras e para isso, as empresas vêm buscando novas alternativas que possam impulsionar o desenvolvimento e a reformulação de práticas no mercado. De acordo com Rodrigues e Veloso (2013), o processo de inovação depende da criatividade e da disposição dos profissionais que atuam nos negócios, já que a criatividade se caracteriza como um conjunto de princípios e ações 
transformadoras de profissionais e do ambiente laboral na busca pela criação de algo original, útil e adequado ao mercado (Braia, Curral, \& Gomes, 2014; Zhou \& Su, 2010).

Para compreender a dimensão criativa no ambiente organizacional é necessário considerar os aspectos que incentivam a criação e influenciam o desenvolvimento das práticas profissionais inovadoras. Desta maneira, o capital social apresenta-se como elemento propulsor e mediador da relação criatividade-inovação, já que possibilita a troca de informações, construção do conhecimento e interações sociais entre os atores relevantes da empresa, possibilitando e influenciando a implementação de práticas inovadoras (Santos, Vasconcelos, Cândido, \& Caldas, 2013).

Os estudos sobre inovação compreendem diversas perspectivas, formuladas com base na evolução dos negócios frente aos contextos sociais, econômicos e políticos (Bruno-Faria \& Fonseca, 2014). As pesquisas que focam a inovação em bens/serviços caracterizam-se pela compreensão das ações e etapas que permeiam a inserção da inovação para o processo produtivo, seja na criação ou reinvenção de produtos/serviços para o mercado. Já os estudos que se centram no comportamento inovador dos profissionais buscam analisar as atitudes inovadoras, que envolvem desde a identificação e exploração de oportunidades, o gerenciamento de novas ideias (aspecto criativo), assim como também comportamentos que refletem em mudanças, aplicação de novos conhecimentos ou melhorias em processos para desempenho pessoal/profissional (Jong \& Hartog, 2008; Pinheiro, 2012).

Para esta pesquisa focou-se na inovação centrada no sujeito, visando compreender os aspectos subjetivos que podem influenciar no comportamento inovador dos trabalhadores nas organizações. Para à organização, considerada lócus da criação útil, torna-se necessário que a cultura organizacional apoie e incentive o indivíduo para o desenvolvimento de suas práticas, já que é nesse ambiente que se constroem as emoções, motivações e expectativas, em diferentes valências e intensidades. Assim, a motivação do trabalhador para conduta inovadora não pode se tratar como uma atitude isolada, mas sim integrada aos processos e políticas da empresa (Engelman, 2016).

Neste contexto, acredita-se que o suporte organizacional seria uma das variáveis que poderiam contribuir quanto a necessidade de compreensão dos aspectos subjetivos do "ser profissional" sob a ótica econômica unidirecional e da inovação, originado das novas perspectivas de gestão, com foco nos processos de interação humana na relação organização-trabalhador, seu desempenho profissional e produtividade (Formiga, Freire, Batista, \& Estevam, 2017; Scorsolini-Comin, Inocente, \& Miura, 2012; Tamayo \& Paschoal, 2003; Tractenberg, 1999).

Este construto (isto é, o suporte organizacional) contribui para a relação organização-trabalhador, por meio da perspectiva de reconhecimento e valorização da atuação profissional (Kurtessis, Eisenberger, Ford, Buffardi, Stewart, \& Adis, 2015). De acordo com os autores supracitados, ao existir uma percepção favorável do suporte, esta, é capaz de fortalecer o envolvimento afetivo do empregado com a organização, resultando em maior esforço do funcionário para atingir os objetivos traçados pela empresa (Oliveira-Castro, Pilati \& Borges-Andrade, 1999).

Um estudo pioneiro referente ao suporte organizacional, a qual, tinha por objetivo verificar aspectos relacionados (a) formação das crenças dos funcionários acerca das ações de valorização que a empresa praticava, (b) redução do absenteísmo, (c) relações entre suporte organizacional percebido e o absenteísmo. Para Eisenberger, Huntington, Hutchison e Sowa (1986), os trabalhadores constroem reflexões e perspectivas acerca do reconhecimento e valorização atribuída pela organização sobre as práticas profissionais, as quais, capaz de influenciar no compromisso profissional e na construção do vínculo afetivo entre o trabalhador e a empresa.

Para isso, estes autores, desenvolveram uma Escala para avaliar a Percepção de Suporte Organizacional (EPSO) e que foi adaptada e validada para o contexto brasileiro (Siqueira, 1995), revelando indicadores psicométricos consistentes, e recentemente, confirmada (Formiga, Freire, \& Fernandes, 2019). Trata-se de um instrumento influente nos fatores psicossociais e comportamentais do profissional, intervindo em aspectos relacionados ao desempenho no trabalho, ao 
comprometimento, cidadania organizacional, rotatividade, engajamento, exaustão no trabalho e autoconceito profissional (Eisenberger, Huntington, Hutchison, \& Sowa, 1986; Byrne I\& Hochwarter, 2008; Jain, Giga, \& Cooper, 2013; Formiga, Freire, Estevam, Fleury, \& Sousa, 2018).

Associado ao suporte organizacional, historicamente, a perspectiva inicial dos estudos sobre capital social elucida as relações construídas em torno da família, das instituições sociais, e do convívio público nas sociedades antigas como instrumento valioso para compartilhamento de recursos, construção de parcerias e identificação do sujeito na convivência social (Coleman, 1988; Burt, 1992; Baker, 1990; Putnam, 1993). Na perspectiva organizacional, Nahapiet e Ghoshal (1998) abordam o capital social como instrumento que permite o desenvolvimento de redes de comunicação e parcerias, e possibilitam a criação e troca de conhecimento. Pode ser compreendido com base em três dimensões, na perspectiva estrutural (vínculo social), relacional (confiança baseada em afetos) e no aspecto cognitivo (objetivos compartilhados) (Zhao, Tang, Liu, \& Liu, 2016).

Nos últimos dois anos, pesquisadores brasileiros (cf. Formiga, Freire, \& Fernandes, 2019), adaptaram e validaram a medida de capital social proposta por Nahapiet e Ghoshal (1998); estes autores, analisaram a qualidade psicométrica da escala de capital social, a partir de uma análise fatorial exploratória, visando verificar a distribuição item-fator e fidedignidade da medida, de forma à avaliar os índices de confiabilidade e validade da escala para aplicação com trabalhadores no contexto brasileiro contemporâneo. Os resultados obtidos pelos autores destacaram a qualidade da escala, revelando fidedignidade e confiança para aplicação em pesquisa nacional.

De forma geral, o capital social destaca-se como recurso valioso e original, já que se caracteriza como o conjunto de relações, vivências, segurança e preceitos que influenciam no comportamento assertivo para o compartilhamento de conhecimento, facilitando a colaboração, interação e difusão de ideias (Aribi \& Dupouët, 2015; Felício, Couto, \& Caiado, 2014; Subramaniam \& Youndt, 2005; Anklam, 2002). Desta maneira, no contexto nacional, observou-se que são poucas as pesquisas que buscam contemplar a inovação centrada no sujeito, em especial, raros os instrumentos que mensuram as atitudes inovadoras nas organizações.

No entanto, destaca-se o estudo de Fandiño, Formiga e Menezes (2018), validado por Formiga, Freire e Fernandes (2020), os quais, validaram uma medida capaz de mensurar a atuação inovadora de trabalhadores frente aos processos organizacionais, obtendo com isso, indicadores psicométricos que garantiram a qualidade desta escala, assim como, a sua influência no suporte organizacional e satisfação profissional em trabalhadores que atuam em empresas públicas e privadas no Rio Grande do Norte.

Desta maneira, a presente pesquisa, tem por objetivo verificar a influência do suporte organizacional no capital social e nas atitudes inovadoras dos profissionais que atuam nas organizações. Para isso, pretende-se analisar de que forma as ações de reconhecimento e valorização da organização em resposta a atuação do profissional, pode influenciar na construção de uma rede de confiança, e assim consequentemente impactar no comportamento inovador do trabalhador na rotina organizacional. Assim, acredita-se responder que o suporte organizacional está positivamente relacionado ao capital social e as atitudes inovadoras; e, quanto maior for a percepção do suporte organizacional, maior será o capital social, bem como, as atitudes inovadoras.

\section{Metodologia}

\section{Amostra}

Este estudo foi do tipo descritivo, exploratório e correlacional, assumindo uma natureza quantitativa, com os trabalhadores de organizações públicas e privadas em Natal, no Rio Grande do Norte. Para calcular a amostra, utilizou-se o pacote estatístico G Power 3.1; software este, destinado à análise do poder estatístico (isto é, o teste de hipótese), tendo como 
base, não apenas o 'n' necessário para a pesquisa, mas, também, o tipo de cálculo a ser realizado (Faul, Erdfelder, Lang \& Buchner, 2007).

Para a coleta de dados deste estudo, considerando uma probabilidade de $95 \%$ ( $\mathrm{p}<0,05)$, magnitude do efeito amostral $(\mathrm{r} \geq 0,30)$ e um padrão de poder hipotético $(\pi \geq 0,80)$ para verificar a significância da amostra. Com isso, observou-se que uma amostra de aproximadamente, 180 sujeitos, revelou-se suficiente para esta pesquisa, tendo como indicadores: $\mathrm{t} \geq 1,98 ; \pi \geq$ 0,94; $\mathrm{p}<0,05)$.

Para o critério de inclusão foi considerado as seguintes características: ter idade acima de 18 anos, estar empregado por mais de um ano, e ser ativo no seu setor de trabalho (seja prestador de serviço ou CLT). Essa pesquisa caracteriza-se como do tipo intencional, pois considerou-se a pessoa que, consultada através do e-mail ou rede social, se dispusera a colaborar respondendo o questionário. Desta maneira, uma amostra com 200 trabalhadores na cidade de Natal - RN, com a maioria (51\%) na faixa etária de 30 a 44 anos, sendo $53 \%$ de homens e $47 \%$ de mulheres, tendo $52 \%$ trabalhando no setor público e $56 \%$ tendo uma renda acima de 4.001,00.

\section{Coleta de Dados}

Seguindo as recomendações de Pereira et. al. (2018) utilizou-se o questionário visando o alcance de muitos participantes, para o qual, permite abonar o anonimato dos retornos e a influência de julgamentos do pesquisador. Assim, a coleta foi realizada por meio de questionários eletrônicos inseridos na plataforma de acesso google forms e aplicados enviando por e-mail e compartilhado em redes sociais. Buscou-se a participação voluntária dos sujeitos, informando que não era necessária a identificação e que poderiam desistir da pesquisa no momento que quisessem, não havendo prejuízo algum para eles. O questionário foi disponibilizado durante o mês de junho e um tempo máximo de 40 minutos foi suficiente para que estes realizassem a tarefa. Foram adotados os procedimentos éticos para pesquisas com seres humanos, os quais, seguiram as orientações previstas na Resolução 196/96 do CNS e na Resolução 016/2000 do Conselho Federal de Psicologia (CNS, 1996; ANPEPP, 2000).

As pessoas que se mostraram interessadas e deram o seu consentimento em participar para fazer parte da amostra do estudo, quando acessava o link, apresentava-lhe uma introdução informando sobre os seguintes pontos: as suas respostas serão pessoais e sem interferência do administrador da pesquisa, ao responder o instrumento; não haveria respostas certas ou erradas e elas serão tratadas de acordo com o que o sujeito pensou ao ler as questões apresentadas e como indicou a sua resposta no instrumento apresentado; assegurou-se que as questões respondidas seriam tratadas em seu conjunto de resposta e não na particularidade da resposta de cada sujeito. Apesar das instruções necessárias para que o questionário serem encontrados no próprio link da pesquisa, o pesquisador (com experiência prévia na pesquisa) estava disponível (através do e-mail), caso o respondente tivesse necessidade de esclarecimento sobre as dúvidas que surgirem.

\section{Instrumentos de Pesquisa}

O questionário da pesquisa foi composto por três medidas sobre o comportamento organizacional:

A Escala de Percepção de Suporte Organizacional (EPSO) trata-se de uma escala composta por 9 itens, desenvolvida por Eisenberger et al. (1986) adaptada e validada inicialmente para o contexto brasileiro por Siqueira (1995), em que atualmente destaca-se o estudo de Formiga, Freire e Fernandes (2019). Esta medida tem por objetivo avaliar o quanto as pessoas (que trabalham em organizações públicas e/ou privadas) percebem que a empresa tem uma preocupação com o bemestar do empregado. Os estudos brasileiros recentes que utilizam e validam a medida referem-se as pesquisas propostas por Formiga e Souza, 2014 (Formiga, Fleury \& Souza 2014; Formiga, Bassanin, Souza, Silva, \& Souza 2015; Fleury, Formiga, Souza, \& Souza, 2017; Formiga, Miranda, Araújo, Pastana \& Mafra, 2018), com trabalhadores em diferentes organizações na 
região nordeste e sudeste, no qual revelam que a EPSO, não apenas apresenta alfas próximos ao observado por Siqueira (1995), mas, através da análise fatorial confirmatória, apontam que a estrutura fatorial original vem revelando indicadores psicométricos confiáveis.

A Escala de Capital Social foi criada por Nahapiet e Ghoshal (1998) e validada por (Formiga, Freire \& Fernandes, 2019) para o contexto nacional. Formada por 25 itens, propõe-se identificar a existência de vínculos entre os profissionais da organização, os tipos de relacionamentos construídos e a percepção dos trabalhadores acerca da importância dessa interação, conexão, e compartilhamento de experiências. O modelo utilizado corresponde ao instrumento validado Formiga, Freire e Fernandes (2019), numa amostra de trabalhadores de organizações públicas e privadas, no qual revelou a existência de indicadores estatísticos correspondentes aos exigidos na literatura relacionada a psicometria, considerando a escala confiável e fidedigna.

A Medida de Inovação refere-se a escala proposta no estudo de Formiga, Freire e Fernandes (2020), em que foi adaptada do modelo original de Chang e Liu (2008). Caracteriza-se pela presença de 6 afirmativas que buscam avaliar o comportamento inovador dos trabalhadores em relação as atividades exercidas durante a rotina do profissional na organização. Para mensurar os constructos, o respondente deveria indicar a sua resposta, marcando com um X ou clicando na opção desejada, em uma escala de sete pontos que varia de $1=$ discordo totalmente, $2=$ discordo bastante, $3=$ discordo pouco, $4=$ nem concordo e nem discordo, $5=$ concordo pouco, $6=$ concordo bastante, $7=$ concordo totalmente. Além destes instrumentos, os sujeitos responderam também alguns dados sociodemográficos, relacionados a idade, sexo, escolaridade, renda econômica, setor profissional, região em que reside, e tempo de serviço.

\section{Tratamento dos Dados}

Os dados foram analisados através do software SPSS (versão 24.0), calculando, além de estatísticas descritivas (média, desvio padrão, frequência), teste t de Student, correlação de Pearson e Manova.

\section{Resultados e Discussão}

Após a coleta de dados, avaliaram-se a existência de dados omissos no estudo, estes, estiveram abaixo de 5\% definido por Tabachnick e Fidell (2001), como adequado, podendo não interferir nas análises estatística do estudo. Quanto a multicolinearidade entre as variáveis, as correlações entre elas atenderam aos parâmetros definidos por Tabachnick e Fidell (2001) [ $r \leq 0,90$, variando de 0,43 a 0,72], resultados estes, que permitem gerar modelos com baixo erro. Em relação aos outliers multivariados na amostra, o teste de normalidade de Kolmogorov-Smirnov (KS), revelou normalidade (KS = 0,91; $<<$ 0,18). Por fim, em relação a variância comum do método (common method variance), através do teste de um fator de Harman, a amostra revelou um único fator, com autovalor acima de 1.00, explicando menos de 50\% (40,90\%) da covariância nas variáveis, condição que implica a não existência de um problema de variância comum do método (Podsakoff, Mackenzie, Lee \& Podsakoff, 2003).

Com base nestes resultados, atendeu-se ao objetivo principal do estudo; para isso, realizou-se uma correlação de Pearson (r) entre as variáveis tendo observado, na Tabela 1, a existência de uma relação positiva, forte e significativa, entre todas as variáveis, as quais, foram acima de 0,30 . 
Tabela 1 - Análise descritiva e correlação de Pearson (r) do suporte organizacional, capital social organizacional e atitudes de inovação em trabalhadores de distintas organizações.

\begin{tabular}{c|c|c|c|c|c|c}
\hline $\begin{array}{c}\text { Variável } \\
\text { independente }\end{array}$ & Média & d.p. & Min-Máx & \multicolumn{2}{|c}{$\begin{array}{c}\text { Correlação } \\
\text { entre } \\
\text { variáveis (r) }\end{array}$} \\
\hline $\begin{array}{c}\text { Percepção Suporte } \\
\text { Organizacional } \\
\text { (PSO) }\end{array}$ & 29,43 & 6,72 & $5,00-35,00$ & ---- & & PSO \\
\hline $\begin{array}{c}\text { Capital Social } \\
\text { Organizacional } \\
(\text { CSO) }\end{array}$ & 134,27 & 27,69 & $33,00-175,00$ & $0,67 *$ & ---- & \\
\hline $\begin{array}{c}\text { Inovação } \\
\text { (INOV) }\end{array}$ & 32,05 & 7,86 & $8,00-42,00$ & $0,58^{*}$ & $0,80^{*}$ & --- \\
\hline
\end{tabular}

Notas: * p-valor <0,001. Fonte: Autores.

Um resultado complementar que poderá ser destacado ao avaliar a relação das dimensões do CSO (dimensão relacional, estrutural e cognitiva) e as demais variáveis; observou-se a existência de uma relação positiva da dimensão do capital social estrutural com PSO e INOVA (respectivamente, (r) 0,71 e 0,76, p < 0,01); no capital social cognitivo houve uma correlação positiva com PSO $(r=0,53, p<0,01)$ e INOVA $(r=0,71, p<0,01)$ e, por fim, uma relação positiva entre o capital social relacional e PSO e INOVA (respectivamente, (r) 0,64 e 0,78, p < 0,01).

A partir dessas relações, efetuou-se uma análise multivariada (Anova), associada ao teste post-hoc de Sheffé, pois, pretendeu avaliar as diferenças nas pontuações médias nas variáveis dependentes (CSO e INOVA) versus independente (PSO); é preciso salientar que as variáveis (independentes e dependentes), correspondente ao somatório dos itens de cada teste, foi dividida em 3 categorias (baixo, moderado e alto), para isso, teve em conta a análise de quartis. Sendo assim, os resultados foram os seguintes (ver Tabela 2): observou-se a existência de resultados significativos no efeito direto e de interação; no capital social e na inovação, individualmente, os escores médios dos respondentes foram mais alto (isto é, na avaliação dos sujeitos, a organização investe no capital e nas atitudes inovadoras); já no efeito de interação confirmou o que se espera, quanto maior a percepção do suporte organizacional, maior o capital social, maior a inovação.

Tabela 2 - Escores médios e a diferença obtida entre o capital social e a inovação, em função do suporte organizacional.

\begin{tabular}{|c|c|c|c|c|c|c|c|c|c|}
\hline \multirow{2}{*}{\multicolumn{2}{|c|}{ Variáveis }} & \multicolumn{3}{|c|}{ Nível } & \multirow[b]{2}{*}{$F$} & \multirow{2}{*}{$\mathrm{OP}$} & \multirow{2}{*}{$\begin{array}{c}\mathrm{d} \\
\text { Cohen }\end{array}$} & \multirow{2}{*}{$\begin{array}{l}\text { Tamanho } \\
\text { do efeito }\end{array}$} & \multirow{2}{*}{$\begin{array}{c}\text { Diferenças } \\
\text { entre os } \\
\text { escores }\end{array}$} \\
\hline & & B & M & A & & & & & \\
\hline \multicolumn{2}{|l|}{$\begin{array}{c}\text { Capital Social } \\
\text { Organizacional }\end{array}$} & $19,40 \pm 1,05$ & $22,01 \pm 0,71$ & $25,49 \pm 1,16$ & $7,57^{*}$ & 0,94 & 10,70 & 0,98 & $\begin{array}{c}\text { Alto suporte } \\
\text { vesus alto } \\
\text { capital } \\
\text { social* } \\
\end{array}$ \\
\hline \multicolumn{2}{|l|}{ Inovação } & $19,47 \pm 1,71$ & $23,26 \pm 0,71$ & $24,15 \pm 1,04$ & $5,10^{*}$ & 0,82 & 7,21 & 0,96 & $\begin{array}{c}\text { Alto suporte } \\
\text { vesus alta } \\
\text { Inovação* }\end{array}$ \\
\hline $\begin{array}{c}\text { Capital Social } \\
\text { Organizacional } \\
\text { versus } \\
\text { Inovação }\end{array}$ & B & $\begin{array}{l}16,96 \pm 0,75 \\
19,80 \pm 1,40 \\
21,67 \pm 3,13 \\
\end{array}$ & $\begin{array}{l}20,00 \pm 1,45 \\
22,58 \pm 0,90 \\
27,22 \pm 1,27 \\
\end{array}$ & $\begin{array}{l}21,25 \pm 2,71 \\
23,64 \pm 1,32 \\
28,85 \pm 0,77 \\
\end{array}$ & $3,14^{*}$ & 0,80 & 4,44 & 0,91 & $\begin{array}{l}\text { lto suporte } \\
\text { vesus alto } \\
\text { capital social } \\
\text { vesus Alta } \\
\text { Inovação }\end{array}$ \\
\hline
\end{tabular}

Notas: $\mathrm{B}=$ Baixo, $\mathrm{M}=$ Moderado, $\mathrm{A}=$ Alto. ${ }^{*} \mathrm{p}$-valor $<0,01 ; \mathrm{R}_{\text {ajustado }}=0,35$. Fonte: Autoria própria. 
Com base nestes resultados, voltou-se para a análise dos tamanhos do efeito (TDE), a qual, foi realizado através do cálculo do 'd' de Cohen; a necessidade dessa avaliação se deve a importância de verificar o TDE, compreendido em termos do grau ou dimensão em que o fenômeno está presente na população ou amostra pesquisada, sendo exclusivo desta. O poder do teste, por sua vez, se refere a suposição de probabilidade do pesquisador em relação ao poder de detecção do teste em sinalizar que a diferença observada na comparação entre os grupos ou eventos avaliados, para poucas variáveis ou sujeitos, observou-se uma significância; para isso, o poder deste teste, em geral, é fixado por convenção, exigindo ser $\geq 80 \%$, em cada 'd' observado (cf. Cohen, 1991a; 1992b). Desta maneira, observou-se um TDE acima de 0,90, podendo afirmar que é possível que tais resultados, de fato, estejam presentes nas organizações, na concepção dos funcionários.

\section{Discussão}

Neste artigo, no objetivo central tratou-se de verificar a relação entre suporte organizacional, capital social organizacional e inovação em trabalhadores nas cidade de Natal no estado do Rio Grande do Norte, tendo seu interesse pautado no tema devido a baixa produção cientifica a respeito da associação entre estas variáveis (Fandiño, Formiga \& Menezes, 2018) e por ter encontrado apenas estudos que contemplam tais variáveis de forma isolada (Siqueira, 1995; Nahapiet \& Ghoshal, 1998; Fandiño, Formiga \& Menezes, 2018), pois, pressupõem que uma avaliação nesta direção é capaz de gerir benefício no desenvolvimento organizacional e na produtividade funcional.

Com esses achados pode-se destacar que as relações observadas entre as variáveis, chama atenção para a intensidade do escore correlacional (o qual, $\geq 0,30$ ), pois, o seu percentual de explicação relacional esteve, aproximadamente, entre $33 \%$ a 64\% (ver tabela 1). Nesta mesma tabela, os escores médios de cada variável estiveram acima da mediana e/ou bem próximos a pontuação do escore máximo, permitindo com isso, refletir que, a partir desses resultados, o processo de trabalho com foco na inovação, não poderá ter sua avaliação, exclusiva, numa forma individual (foco no trabalhador) ou organizacional (foco na empresa e sua dinâmica de investimento laboral), é preciso observar que, na organização, existe um processo, e que ele, não poderá ser polarizado quanto pretensa solução para a motivação de um funcionário inovador e criativo.

Para esta condição compreende-se que essas situações ocorrem sob via de mão dupla; se numa perspectiva, é a empresa que deve valorizar, em outra, faz-se necessário a clareza e implementação de vínculos entre os profissionais da organização, a construção dos tipos de relacionamentos e a percepção dos trabalhadores acerca da importância dessa interação, conexão, e compartilhamento de experiência. Especificamente, para uma funcionalidade inovadora na organização, é preciso o desenvolvimento de uma cultural organizacional, mas, com maior foco na cooperação, mesmo venha sugerir a competitividade.

Ao se investir em modelo de gestão proto-coletivista e/ou proto-individualismo (Gouveia, Andrade, Jesus, Meira, Maja \& Formiga, 2002), o qual preza pelos contatos de orientação para uma melhor competitividade e relacionamento com foco na produtividade e inovação, não se está apenas estimulando uma formação de redes sociais, simplesmente, para manter um relacionamento auto-protecionista, o qual, inibidor de maior produtividade organizacional e estruturação da saúde laboral (Valença, 2017). O processo e dinâmica no ambiente de trabalho, com base nestes resultados, preciso de interação, mas, também, exige atitude única de cada trabalhador, mesmo que ela venha exigir apoio da própria organização e seus gestores; mas, e quando atitude funcional e apoio organizacional for aceita e assimilar por parte do recurso humano? Inova-se! Produz! Compete! Sendo esta condição, uma exclusividade do trabalhador ser proto-individualista, atendendo a meta exigida e o cumprimento do objetivo estabelecido.

De forma geral, estes resultados são importantes por contribuir para uma atividade mais contemporânea frente as exigências inovadoras do recurso humano $(\mathrm{RH})$ e a política organizacional de inovação e competitividade no trabalho; para tanto, no uso dos instrumentos organizacionais do tipo apresentado neste estudo, permitiria verificar a existência deste 
problema-situação. Por meio da avaliação da qualidade do suporte organizacional que o funcionário percebe da empresa, é bem possível que seria capaz de identificar qual melhor capital social para investir nos funcionários quando exige-se inovar.

Ao avaliar a ANOVA, apresentada na tabela 2, ficou notório, considerando os escores médios entre as variáveis, que quanto maior o suporte organizacional, maior o capital social e a inovação, tendo esses resultados, apresentando um TDE significativo garantindo uma aproximação interpretativa hipótese-realidade de pesquisa (isto é, na afirmação de Cohen (1992b), o TDE representa o quanto o fenômeno avaliado é exclusivo da população ou amostra pesquisada).

Desta maneira, os resultados obtidos evidenciam que, provavelmente, o suporte organizacional poderia ser considerado uma variável mediadora frente a atuação e desempenho no ambiente de trabalho, já que influencia em aspectos relacionados a construção dos laços sociais, bem como, na atuação inovadora no âmbito corporativo. (Spector, 2002; Wagner Iii \& Hollenbeck, 2000; Kanaane, 2011; Zanelli; Borges-Andrade \& Bastos, 2004; Siqueira, 2009). As ações de reconhecimento e valorização realizadas pelas organizações, relacionadas as manifestações de aprovação, elogio, retribuição material e social ao esforço dos recursos humanos influenciam diretamente na conduta profissional, motivando o sujeito a desenvolver atitudes inovadoras de forma contínua e construir laços sociais que incentivam o alcance dos objetivos traçados na organização (Oliveira-Castro, Pilati \& Borges-Andrade,1999).

No Brasil, a pesquisa pioneira de Siqueira (1995) contribui muito para refletir os resultados obtidos no presente estudo, pois os achados iniciais identificaram que o suporte organizacional influencia a percepção do trabalhador acerca do compromisso com a organização, já que o profissional ao identificar com essas ações mostra-se comprometido e engajado para atuar em suas atividades rotineiras (Siqueira, 2003). Em direção semelhante, os estudos de Akgunduz, Alkan e Gök (2018; Estivalete et al, 2016; Fernandes, Siqueira \& Vieira, 2014; Celep \& YIlmazturk, 2012), retratam que a variável apresenta relação significativa no desenvolvimento da criatividade, bem-estar no trabalho, compromisso organizacional e até, segundo Altinoz et al, (2016; Karatepe, 2015) a diminuição dos sintomas referente as doenças ocupacionais, relacionadas a síndrome de burnout e o esgotamento emocional.

Desta maneira, a medida de capital social proposta por Nahapiet e Ghoshal (1998) permite o desenvolvimento de interações sociais e construção de parcerias, por meio de três dimensões (estrutural, relacional e cognitiva), a construção e fortalecimento dos laços sociais no ambiente empresarial promovem a interação entre indivíduos, desenvolvimento de sentimentos e o compartilhamento de recursos, sejam eles tangíveis ou intangíveis (Bharati; Zhang; Chaudhury, 2015), os quais, contribuiriam tanto para atitudes inovadoras quanto na produtividade organizacional.

Sendo assim, a relação entre suporte organizacional e capital social demonstra que o trabalhador ao identificar as ações sinceras de reconhecimento e valorização da organização para com a sua atuação profissional constrói uma relação de confiança que incentiva o bem-estar por meio da cooperação e apoio mútuo entre os pares, possibilitando que os profissionais compartilhem conhecimentos, experiências e objetivos (D'araújo, 2003; Zhang, Liu, Chen \& Gong, 2017), os quais, contribuem para a produtividade.

Além disso, nos estudos de Stoeckicht e Soares (2010), o capital social apresenta relação significativa com a capacidade de inovação das empresas, contribuindo para a sobrevivência e sustentabilidade no mercado competitivo. Especificamente, um comportamento inovador caracteriza-se pelo desenvolvimento de atitudes relacionadas ao trabalho em equipe, acesso à educação, conhecimento, TI, assim como ações criativas (Standing, Jackson, Larsen, Suseno, Fulford \& Gengatharen, 2016), condições, as quais, corroboram os achados nesta pesquisa.

Os resultados observados no estudo, convergem para pesquisa de Engelman (2016; Shukla \& Singh, 2015); de acordo com os autores supracitados, os profissionais para atuarem de forma inovadora necessitam estarem vinculados a um ambiente com uma cultura organizacional formada por fatores relacionados a atitude inovadora, inovação incremental, flexibilidade e 
comunicação aberta, adaptabilidade de funcionário, espaço de geração de ideia, abordagem tradicional, estratégias de orientação para resultados, os quais, são capaz de aprimorar competências, motivação para inovar e suporte organizacional.

De forma geral, as empresas que procuram investir mais na compreensão das necessidades dos profissionais, desenvolvimento de uma cultura organizacional voltada para ações de reconhecimento e valorização humana, possuem maior vantagem competitiva no mercado, já que os trabalhadores se motivam quando alcançam os objetivos organizacionais, contribuindo assim para o sucesso da empresa. Sendo assim, um profissional ao se sentir reconhecido por suas práticas, constrói laços de confiança e interações entre pares, desenvolvendo sentimentos pela empresa/função que atua, isto é, reconhece o suporte que sua organização oferece.

Com a implementação de políticas organizacionais incentivadora do suporte organizacional e o capital social, provavelmente, estimulariam na avaliação e implementação de uma cultura no ambiente corporativo dirigida a cooperação, conduzindo o trabalhador para atitudes criativas, autônomas e proativas destinadas a gestão da complexidade do mercado, gerando e gerindo uma conduta pautada em atitudes inovadoras e persistentes na construção e implementação de ideias no ambiente organizacional (Audenaert, Vanderstraeten \& Buyens, 2017). Condições estas, que contribuiria para uma gestão à avaliação e implementação de programa de formação, intervenção e treinamento, destinados a gestão de profissionais mais socialmente culturalizados, criativos e com uma autogestão.

\section{Considerações Finais}

Em termo gerais, o estudo possibilitou verificar a importância da implementação e desenvolvimento de uma cultura organizacional voltada para a cooperação, tendo por base de avaliação, a percepção do funcionário. Os profissionais ao atuarem em organizações que estimulam ações de reconhecimento e valorização das práticas profissionais, incentivam a construção de relacionamentos, interações, conexões e compartilhamento de experiências entre sujeitos, motivando o indivíduo a atuar de forma criativa e inovadora. Dessa forma, o incentivo ao capital social no meio empresarial possibilita o compartilhamento de recursos entre empresas, entre organização-mercado, bem como, de experiências e sentimentos vividos na organização e suas demandas de gestão entre os profissionais (Bharati, Zhang \& Chaudhury, 2015).

Apesar desses resultados serem aceitáveis e os objetivos propostos alcançados, algumas direções deverão ser considerando para estudos futuros: avaliar a relação entre suporte organizacional, capital social e inovação em trabalhadores que atuam em empresas públicas e privadas com foco transcultural; avaliar os diferentes contextos políticos, sociais e econômicos em relação as políticas implementadas no ambiente corporativo; inserir variáveis que verificasse o tipo e intensidade cultural referente a organização das empresas e o quanto ela poderia influenciar no suporte, capital social e inovação. Também, seria muito benéfico, no campo do recurso humano, um investimento de pesquisa e intervenção na área da psicologia organizacional e do trabalho, ressaltando mais pontos saudáveis do trabalhador.

\section{Referências}

Altinoz, M., Cop, S., Cakiroglu, D. \& Altinoz, T. (2016). The Influence of Organization Support Perceived in Enterprises on Burnout Feeling: A Field Research. Procedia-Social and Behavioral Sciences, 235(1), 427-43.

Akgunduz, Y., Alkan, C. \& Gok, O. A. (2018) Perceived organizational support, employee creativity and proactive personality: The mediating effect of meaning of work. Journal Of Hospitality And Tourism Management, 34(1), 105-114.

Anklam, P. (2002). Knowledge management: the collaboration thread. Bulletin of the Association for Information Science and Technology, $28(6), 8-11$.

Aribi, A. \& Dupouët, O. (2015). The role of organizational and social capital in the firm's absorptive capacity. Journal of Knowledge Management, 19(5), 9871006,2015

Armstrong, S. J., \& Overton, T.S. (1977). Estimating non-responses bias in mailed surveys. Journal of Marketing Research, 13(1), 396-402. 
Associação Nacional de Pesquisa e Pós-Graduação em Psicologia - ANPEPP. (2000). Contribuições para a discussão das Resoluções CNS no. 196/96 e CFP. No 016/2000.

Audenaert, M., Vanderstraeten, A. \& Buyens, D. (2017) When innovation requirements empower individual innovation: The role of job complexity. Personnel Review, 46(3), 608-623.

Baker, W. E. (1990) Market networks and corporate behavior. American journal of sociology. 96(3), 589-625.

Bharati, P., Zhang, W. \& Chaudhury, A. (2015) Better knowledge with social media? Exploring the roles of social capital and organizational knowledge management. Journal of Knowledge Management, 19(3), 456-475.

Braia, F., Curral, L. \& Gomes, C. (2014). Criatividade em contexto organizacional: o impacto de recompensas extrínsecas e do feedback negativo no desempenho criativo. Psicologia, 28(2), 45-62.

Bruno-Faria, M. F. \& Fonseca, M. V. A. (2014). Cultura de inovação: conceitos e modelos teóricos. RAC-Revista de Administração Contemporânea, 18(4).

Burt, R. S. (1992) The social structure of competition. In: Explorations in Economic Sociology, Russell Sage Foundation.

Byrne, Z. S.; \& Hochwarter, W. A. (2008). Perceived organizational support and performance: Relationships across levels of organizational cynicism. Journal of Managerial Psychology, 23(1), 54-72.

Celep, C. \& Yilmazturk, O. E. (2012). The relationship among organizational trust, multidimensional organizational commitment and perceived organizational support in educational organizations. Procedia-Social and Behavioral Sciences, 46(1), 5763-5776.

Chang, L. \& Liu, C. (2008) Employee empowerment, innovative behavior and job productivity of public health nurses: A cross-sectional questionnaire survey. International journal of nursing studies, 45(10), 1442-1448.

Cohen, J. A. (1992a) Power primer. Psychological bulletin, 112(1), 155.

Cohen, J. (1992b) Statistical power analysis current directions. Psychological Science, 1(3) 98-101.

Coleman, J. S. (1988). Social Capital in the Creation of Human Capital. The American Journal of Sociology, 94(1), 95-120.

Conselho Nacional De Saude - CNS. (2012). Diretrizes e Normas Regulamentadoras de Pesquisas Envolvendo Seres Humanos.

Dancey, C. P. \& Reidy, J. (2006). Estatística sem matemática para psicologia. Artmed.

Eisenberger, R., Huntington, R., Hutchison, S. \& Sowa, D. (1986). Perceived Organizational Support. Journal of Applied, 71(3), 500-50.

Engelman, R. M. \& Gonçalves, M. A. (2016). Emoções, criatividade e inovação: reflexões sobre esta relação. Revista Gestão e Desenvolvimento, 13(2), 37-49.

Estivalete, V. F. B., Andrade, T., Faller, L. P., Stefanan, A. A. \& Souza, D. L. (2016) Suporte social e suporte organizacional como antecedentes do bem-estar no trabalho: a perspectiva de colaboradores de uma empresa de logística ferroviária. Revista de Administração da UNIMEP, 14(2).

Fandiño, A. M., Formiga, N. S. \& Menezes, R. M. P. (2018). Organizational Social Capital, Resilience and Innovation validation of a theoretical model for specialized workers. Journal of Strategy and Management.

Faul, F., Erdfelder, E., Lang, A. G., \& Buchner, A. (2007). G* Power 3: A flexible statistical power analysis program for the social, behavioral, and biomedical sciences. Behavior research methods, 39(2), 175-191.

Felício, J. A., Couto, E. \& Caiado, J. (2014). Human capital, social capital and organizational performance. Management Decision, 52 (2), $350-364$.

Fleury, L. F. O., Formiga, N. S., Souza, M. A. \& Souza, M. A. F. (2017). Escala de Percepção de Suporte Organizacional: evidência da estrutura fatorial em trabalhadores brasileiros. Psicologia em Pesquisa, 11(1), 1-2.

Formiga, N. S., Bassanin, L. L., Souza, M. V., Silva, F. M. C., Da Silva, G. G. E. \& Souza, M. A. (2015). Suporte organizacional em trabalhadores de diferentes empresas: comprovação de uma medida. Revista de Psicología Universidad de Antioquia, 7(1), 9-20.

Formiga, N. S., Freire, B. G. O., Batista, P. F. A. \& Estevam, I. D. (2017) Suporte organizacional e autoestima em funcionários de organizações públicas e privadas no brasil. Psicologia.com.pt, p.1-15.

Formiga, N. S., Freire, B. G. O., Estevam, I. D. Fleury, L. F. O., \&Souza, M. A. (2018). A influência do suporte organizacional no autoconceito profissional em trabalhadores de organizações públicas e privadas. Eureka: Revista de psicologia, 15(1), 78-95.

Formiga, N. S., Freire, B. G. O, \& Bruna Gonçalves De Oliveira; Fernandes, A. (2019). Evidência métrica de construto e invariância fatorial da escala de percepção de suporte organizacional em trabalhadores brasileiros. Revista de Gestão e Secretariado, 10(2), 194-221).

Formiga, N. S., Freire, B. G. O. \& Fernandes, A. (2019). Evidência de construto, invariância fatorial e validade convergente da escala de capital social organizacional em trabalhadores brasileiros. EUREKA, 16(1), 34-59.

Formiga, N. S., Freire, B. G. O. \& Fernandes, A. (2020). A medida da atitude de inovação: valores métricos, invariância fatorial e diferenças em trabalhadores. Revista de Carreiras e Pessoas (ReCaPe), 10(2).

Formiga, N. S., Fleury, L. F. O., Souza, M. A. (2014). Evidências de validade da escala de percepção de suporte organizacional em funcionários de empresa pública e privada. Estudos Interdisciplinares em Psicologia, 5(2), 60-76. 
Formiga, N. S., Miranda, A. L. B. B, Araújo, I. T., Pastana, S. T. G \& Mafra, A. L. (2018). Evidência da invariância fatorial e validade convergente da escala de suporte organizacional: estudo com trabalhadores brasileiros. Boletim-Academia Paulista de Psicologia, 38(94), $27-35$.

Formiga, N. S. \& Souza, M. A. (2014). Comprovação empírica de uma medida psicológica sobre a percepção do suporte organizacional em trabalhadores de diferentes empresas. Boletim Academia Paulista de Psicologia, 34(87), 510-552.

Gable, S. L. \& Haidt, J. (2005). What (and why) is positive psychology? Review of general psychology, 9(2), 103-110.

Gouveia, V. V., Andrade, J. M., Jesus, G. R., Meira, Maja, \& Formiga, Nilton S. (2002). Escala multi-fatorial de individualismo e coletivismo: elaboração e validação de construto. Psicologia: Teoria e Pesquisa, 18(2), 203-212.

Jain, A. K.; Giga, S. I. \& Cooper, C. L. (2013). Perceived organizational support as a moderator in the relationship between organisational stressors and organizational citizenship behaviors. International Journal of Organizational Analysis, 21(3), 313-334.

Jong, J. P \& Hartog, D. N. D. (2008) Innovative work behavior: Measurement and validation. EIM Business and Policy Research, $1(1), 1-27$.

Kanaane, R. (2011). Comportamento humano nas organizações: o homem rumo ao século XXI. (2a ed.), Atlas.

Karatepe, O. M. (2015). Do personal resources mediate the effect of perceived organizational support on emotional exhaustion and job outcomes? International Journal of Contemporary Hospitality Management, 27(1), 4-26.

Kurtessis, J. N., Eisenberger, R., Ford, M. T., Buffardi, L. C., Stewart, K. A. \& Adis, C. S. (2017). Perceived organizational support: A meta-analytic evaluation of organizational support theory. Journal of Management, 43(6), 1854-1884

Miles, Jeremy; Shevlin, Mark. (2001). Applying regression and correlation: A guide for students and researchers. Sage Publications.

Nahapiet, J. \& Ghoshal, S. (1998) Social Capital, Intellectual Capital, and the Organizational Advantage. The Academy of Management Review, 23(2), 242-266.

Oliveira-Castro, G. A., Pilati, R., Borges- Andrade, J. E. (1999). Percepção de suporte organizacional: desenvolvimento e validação de um questionário. Revista de Administração Contemporânea, 3(2), 29-51.

Pasquali, Luiz. (2011). Psicometria: teoria dos testes na psicologia e na educação. (4a ed.), Vozes.

Pavot, W. \& Diener, E. (1993). Review of the satisfaction with life scale. Psychological Assessment, 5(2), 164-172.

Pereira A.S. et al. (2018). Metodologia da pesquisa científica. UFSM.

Pinheiro, V. S. B. (2012). Fatores psicossociais no trabalho e comportamentos inovadores de trabalho. Dissertação de Mestrado, Covilhã

Putnam, R. D. (1993). What makes democracy work?. National Civic Review, 82(2), 101-107, 1993.

Rodrigues, A. F. \& Veloso, A. (2013). Contribuições da gestão de recursos humanos para a criatividade e inovação org anizacional. Revista Psicologia Organizações e Trabalho, 13(3), 293-308

Santos, J. E. A., Vasconcelos, A. C. F., Candido, G. A. \& Caldas, P. T. (2013). Capital Social como Elemento Facilitador do Processo de Inovação: Um Estudo Exploratório com Produtores e Comerciantes de Produtos Agrícolas. Cadernos Gestão Social, 4(1), 57-69.

Scorsolini-Comin, F. Inocente, D. F. \& Miura, I. K. (2012). Avaliação de um programa de treinamento corporativo: Em busca da mudança organizacional. Revista Brasileira de Orientação Profissional, 13(1).

Shukla, T. \& Singh, A. (2015). Organizational Factors Influencing Innovation: An Empirical Investigation. Journal of Strategic Human Resource Management, 4(3).

Silva, R. S., Cappellozza, A. \& Costa, L. V. (2015) O impacto do suporte organizacional e do comprometimento afetivo sobre a rotatividade. Revista de Administração IMED, 4(3). 314-329.

Siqueira, M. M. M. (1995). Antecedentes de comportamentos de cidadania organizacional: a análise de um modelo pós-cognitivo. 198 f. Tese (Doutorado em Psicologia). Universidade de Brasília, Brasília.

Siqueira, M. M. M. (2009). Medidas do comportamento organizacional: ferramentas de diagnóstico e de gestão. Artmed Editora.

Spector, P. E. (2002). Psicologia nas organizações. (2a ed.), Saraiva.

Standing, C., Jackson, D., Larsen, A., Suseno, Y., Fulford, R. \& Gengatharen, D. (2016) Enhancing individual innovation in organisations: a review of the literature. International Journal of Innovation and Learning, 19 (1), 44- 62.

Stoeckicht, I. P. \& Soares, C. A. P. A. (2010). Importância da gestão do capital social para o desenvolvimento da capacidade de inovar em empresas brasileiras. INGEPRO-Inovação, Gestão e Produção, (10), 23-37.

Subramaniam, M. \& Youndt, M. A. (2005). The influence of intellectual capital on the types of innovative capabilities. Academy of Management journal, 48(3), 450-463.

Tabachnick, B. G \& Fidell, L. S. (2001). Using multivariate statistics. Allyn \& Bacon.

Tamayo, A. \& Paschoal, T. (2003). A relação da motivação para o trabalho com as metas do trabalhador. Revista de Administração Contemporânea, 7(4), 3354. 
Research, Society and Development, v. 10, n. 3, e55710313753, 2021

(CC BY 4.0) | ISSN 2525-3409 | DOI: http://dx.doi.org/10.33448/rsd-v10i3.13753

Tractenberg, L. (1999) A complexidade nas organizações: futuros desafios para o psicólogo frente à reestruturação competitiva. Psicologia: ciência e profissão, 19(1), 14-29, 1999.

Verstegen, B. H. (2011). A socio-economic view on management control. International journal of social economics, 38(2), $114-127$.

Wagner, I. J. A. \& Hollenbeck, J. R. (2000) Comportamento organizacional. Saraiva.

Zhang, X. et al. (2017). Social capital, motivations, and knowledge sharing intention in health Q\&A communities. Management Decision, 55(7), 1536-1557.

Zhao, X., Tang, Q., Liu, S. \& Liu, F. (2016). Social capital, motivations, and mobile coupon sharing. Industrial Management \& Data Systems, 116(1), 188206.

Zhou, J. \& Su, Y. (2010). A missing piece of the puzzle: The organizational context in cultural patterns of creativity. Management and Organization Review, 6(3), 391-413. 